\title{
A recipe for targeted therapy in prostate cancer
}

\section{Shigeo Masuda and Juan Carlos Izpisua Belmonte}

In their Review, Marignol and colleagues discuss the comprehensive knowledge of the biology of prostate cancer, which is deeply associated with hypoxia and notch signalling (Marignol, L., Rivera-Figueroa, K., Lynch, T. \& Hollywood, D. Hypoxia, notch signalling, and prostate cancer. Nat. Rev. Urol. 10, 405-413; 2013). ${ }^{1}$ On this topic, an additional three issues related to targeted therapy should be discussed based on recent evidence: the role of tumour angiogenesis, notch signalling inhibition (by $\gamma$-secretase inhibitors [GSIs]) and cancer stem cells.

Firstly, hypoxia is known to be a common feature of prostate tumours, as Marignol and colleagues mentioned. ${ }^{1}$ Interestingly, recent studies ${ }^{2,3}$ have demonstrated that addition of therapy targeting vascular endothelial growth factor (VEGF) to standard of care showed no improvement in the overall survival of patients with metastatic castration-resistant prostate cancer. Specifically, the soluble VEGF decoy receptor (aflibercept) ${ }^{2}$ and anti-VEGF antibody (bevacizumab) ${ }^{3}$ were each added to the docetaxel plus prednisone backbone to inhibit angiogenesis, but resulted in disappointing clinical outcomes. That is, inhibiting angiogenesis in already hypoxic tumours probably does not have an effect.

Second, notch signalling has been shown to be involved in the pathogenesis of prostate cancer, although it remains to be elucidated whether the pathway has an oncogenic or a tumour-suppressive role. ${ }^{4}$ In some cases, the effects of notch signalling on prostate tumorigenesis might be context-dependent, varying between prostate cancers. If notch signalling is activated and has an oncogenic role in a patient with prostate cancer, notch inhibition by GSIs might be effective in a cell-autonomous manner (that is, by directly inducing apoptosis in tumour cells). GSIs inhibit the cleavage of the notch intracellular domain, resulting in the blockade of notch activation. GSIs, which have not yet gained FDA approval for cancer treatment, are being assessed in clinical trials (NCT00100152, NCT00106145,
NCT01088763, NCT01116687). The gastrointestinal toxic effects of GSIs are well known, ${ }^{5}$ but steroid therapy has been suggested to reduce their incidence. A previous study by Real and colleagues ${ }^{6}$ reported that GSI-induced toxicity was reduced by glucocorticoid therapy in mice. Furthermore, in a phase I trial of a GSI in children with brain tumours, Fouladi and colleagues ${ }^{7}$ described that the seven patients who received steroids for other indications did not experience gastrointestinal toxicity. Of the 16 patients who were not taking steroids, two patients experienced gastrointestinal toxicity; as the difference between the two groups was not statistically significant, further prospective studies will be necessary. Intermittent dosing ( 3 days on, 4 days off) of GSI was also recommended to reduce toxicity on the basis of two other studies. ${ }^{8,9}$ Indeed, standard therapy for prostate cancer includes steroids, which will be advantageous for patients taking GSI-combined therapy.

However, GSIs might also act in a non-cellautonomous manner, ${ }^{10}$ modulating processes in the tumour microenvironment, such as tumour angiogenesis. If so, GSIs might induce nonfunctional angiogenesis (abnormal vessels) with low perfusion capacity, ${ }^{11}$ which would lead to hypoxia and might contribute to the enhanced survival of putative prostate cancer stem cells. For patients with prostate cancer, the possibility is that the efficacy of GSI therapy will be weighed against the hypoxia that might occur as a result of nonfunctional angiogenesis, which might eventually promote tumour proliferation. To avoid this scenario, further elucidation of the mechanism of GSI activity in prostate cancer is required.

Finally, notch signalling and hypoxia have been implicated in the maintenance of cancer stem cells. Recent evidence in leukaemic stem cells indicates that even if a drug targeting only leukaemic stem cells is used to treat leukaemia, it might not provide a cure; rather, seemingly normal haematopoietic stem cells (HSCs) should also be targeted to induce cure. ${ }^{12}$ Specifically, Jan and colleagues ${ }^{12}$ identified clonal progression of mutations in HSCs, leading to relapse. Although no evidence of this effect exists in prostate cancer, it should be determined whether the same scenario exists in prostate cancer stem cells, and whether targeted therapy against these cells in particular would be a rational approach in prostate cancer.

In summary, prostate cancer should be considered to be a hypovascular tumour, for which GSI plus steroid might be a potential treatment. Illuminating the properties of putative cancer stem cells in prostate cancer will improve the strategy for targeted therapy.

Gene Expression Laboratory, The Salk Institute for Biological Studies, 10010 North Torrey Pines Road, La Jolla, CA 92037, USA (S.M., J.C.I.B.).

Correspondence to: S.M.

smasuda@salk.edu

\section{Competing interests}

The authors declare no competing interests.

1. Marignol, L., Rivera-Figueroa, K., Lynch, T. \& Hollywood, D. Hypoxia, notch signalling, and prostate cancer. Nat. Rev. Urol. 10, 405-413 (2013).

2. Tannock, I. F. et al. Aflibercept versus placebo in combination with docetaxel and prednisone for treatment of men with metastatic castration-resistant prostate cancer (VENICE): a phase 3 , double-blind randomised trial. Lancet Oncol. 14, 760-768 (2013).

3. Kelly, W. K. et al. Randomized, double-blind, placebo-controlled phase III trial comparing docetaxel and prednisone with or without bevacizumab in men with metastatic castration resistant prostate cancer: CALGB 90401. J. Clin. Oncol. 30, 1534-1540 (2012).

4. Leong, K. G. \& Gao, W. Q. The Notch pathway in prostate development and cancer. Differentiation 76, 699-716 (2008).

5. van Es, J. H. et al. Notch/gamma-secretase inhibition turns proliferative cells in intestinal crypts and adenomas into goblet cells. Nature 435, 959-963 (2005).

6. Real, P. J. et al. Gamma-secretase inhibitors reverse glucocorticoid resistance in T cell acute lymphoblastic leukemia. Nat. Med. 15, 50-58 (2009).

7. Fouladi, M. et al. Phase I trial of MK-0752 in children with refractory CNS malignancies: a pediatric brain tumor consortium study. J. Clin. Oncol. 29, 3529-3534 (2011).

8. Luistro, L. et al. Preclinical profile of a potent gamma-secretase inhibitor targeting notch signaling with in vivo efficacy and pharmacodynamics properties. Cancer Res. 69, 7672-7680 (2009). 


\section{CORRESPONDENCE}

9. Tolcher, A. W. et al. Phase I study of R04929097, a gamma secretase inhibitor of Notch signaling, in patients with refractory metastatic or locally advanced solid tumors. J. Clin. Oncol. 30, 2348-2353 (2012)
10. Masuda, S. et al. Dual antitumor mechanisms of Notch signaling inhibitor in a T-cell acute lymphoblastic leukemia xenograft model. Cancer Sci. 100, 2444-2450 (2009).

11. Ridgway, J. et al. Inhibition of DII4 signalling inhibits tumour growth by deregulating angiogenesis. Nature $\mathbf{4 4 4}$ 1083-1087 (2006).

12. Jan, M. et al. Clonal evolution of preleukemic hematopoietic stem cells precedes human acute myeloid leukemia. Sci. Transl. Med. 4, 149ra118 (2012). 